AIAA-01-1132

Microgravity Propellant Tank Geyser Analysis and Prediction

Randall J. Thornton

NASA - Marshall Space Flight Center

Huntsville, AL

John I. Hochstein

The University of Memphis

Memphis, TN 
AIAA-01-1132

\title{
MICROGRAVITY PROPELLANT TANK GEYSER ANALYSIS AND PREDICTION
}

\author{
Randall J. Thornton ${ }^{*}$ \\ NASA - Marshall Space Flight Center \\ Huntsville, Alabama \\ John I. Hochstein ${ }^{\dagger}$, \\ The University of Memphis \\ Memphis, Tennessee
}

\begin{abstract}
An established correlation for geyser height prediction of an axial jet inflow into a microgravity propellant tank was analyzed and an effort to develop an improved correlation was made. The original correlation, developed using data from ethanol flow in small-scale drop tower tests, uses the jet-Weber number and the jetBond number to predict geyser height. A new correlation was developed from the same set of experimental data using the jet-Weber number and both the jet-Bond number and tank-Bond number to describe the geyser formation. The resulting correlation produced nearly a $40 \%$ reduction in geyser height predictive error compared to the original correlation with experimental data. Two additional tanks were computationally modeled in addition to the small-scale tank used in the drop tower testing. One of these tanks was a $50 \%$ enlarged small-scale tank and the other a full-scale $2 \mathrm{~m}$ radius tank. Simulations were also run for liquid oxygen and liquid hydrogen. Results indicated that the new correlation outperformed the original correlation in geyser height prediction under most circumstances. The new correlation has also shown a superior ability to recognize the difference between flow patterns II (geyser formation only) and III (pooling at opposite end of tank from the bulk fluid region).
\end{abstract}

\section{NOMENCLATURE}

\begin{tabular}{ll} 
& \multicolumn{1}{r}{$\underline{\text { Symbols }}$} \\
$a$ & acceleration \\
Bo & Bond number \\
$D$ & diameter
\end{tabular}

- Mechanical Engineer, Member AIAA

t Mechanical Engineering Dept., Professor / Department Chair, Member AlAA

Copyright $\bigcirc 2000$ The American Instikute of Aeronautics and Astronautics Inc. All rights reserved.

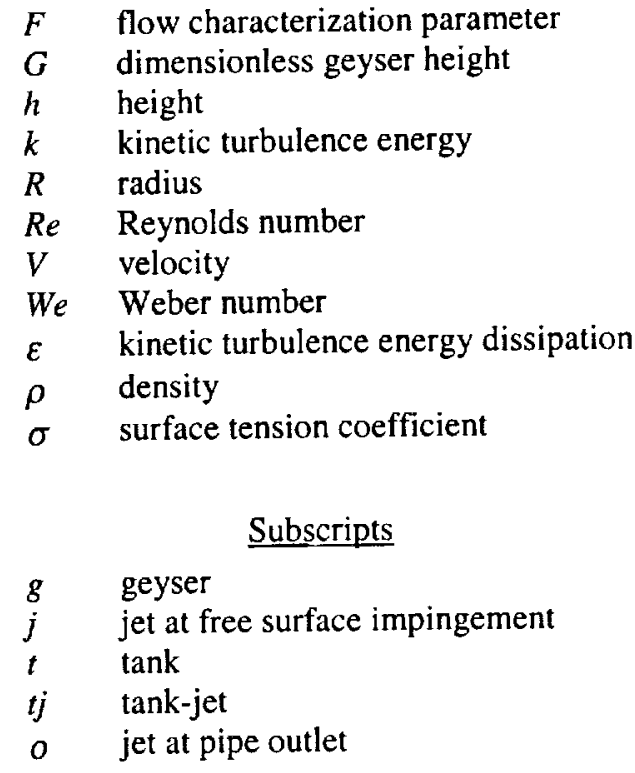

\section{INTRODUCTION}

Most current liquid propulsion systems require the use of cryogenic propellants. The management of these cryogenic propellants in a zero or microgravity environment is crucial for the success of many different types of space missions. Extended duration missions establish the need to store cryogenic fluids such as liquid hydrogen and liquid oxygen in space for long periods either within the vehicle or in orbiting fuel depots. Storing propellants in the extreme environment of space for long periods presents some unique problems.

\section{Propellant Storage Problems}

A major problem in managing a cryogenic propellant is tank self-pressurization. Incident solar radiation heats the cryogenic fluid causing liquid to vaporize and the tank pressure to rise. If this self-pressurization were 
allowed to continue unchecked the tank would rupture. Insulation helps slow radiation from heating the fluid, but pressure build up does eventually occur. A stronger tank is one solution, but this would require an increase in vehicle mass when a lightweight tank design is preferable. Another possible solution is tank venting. The tank venting method is undesirable because it wastes valuable propellant. With launch costs on the order of $\$ 10,000$ per pound, any wasted propellant means a significant loss of money and in orbit resources. In addition, the lack of gravity to positively orient the propellant in a predictable manner makes it nearly impossible to locate a vent where it could be certain that only vapor would be vented. In fact, even in partially filled tanks, it is possible that the entire tank surface could be wetted due to the influence of surface tension in a microgravity environment. Venting is currently the most popular solution, but venting designs must employ thrusters that create an acceleration large enough to ensure that fluid is collected at the end of the tank opposite from the vent. These thrusters add mass to the spacecraft, as does their propellant. In addition, the acceleration created during the re-orientation process introduces the possibility of on-board operations disruption or altering the vehicle's flight path. Thus, an alternative solution avoiding these problems is desirable.

\section{Thermodynamic Vent System}

An alternative solution to the tank self-pressurization problem is a Thermodynamic Vent System, or TVS ${ }^{1,2,3}$ (Figure 1). The TVS would extract a small portion of the bulk liquid from the tank and pass it through a Joule-Thomson valve. The Joule-Thomson valve produces a reduction in temperature as well as pressure in the fluid. Once cooled, the fluid would be routed through a heat exchanger, which would be used to cool a separate flow of propellant also extracted from the bulk liquid. If the fluid leaving the Joule-Thomson valve is a two-phase mixture, it will continue changing phase in the heat exchanger until it is completely vaporized and will eventually be sacrificially vented overboard. The separate stream of cooled liquid would be pumped from the heat exchanger back into the tank and injected through an axial jet pipe located at the fore end of the tank. The fore end of the tank is where the bulk fluid should be in an earth-orbiting mission. Although minimal at the altitudes that spacecraft orbit the earth, aerodynamic drag on the spacecraft is large enough to orient the bulk fluid in the fore end of the tank, thus the location of the bulk fluid is known. However, this does not in any way give a positive location for a dry wall for venting due to surface tension effects in a microgravity environment.

\section{Jet Flow Benefits}

The jet flow provides several benefits including mixing of the bulk liquid. The mixing action helps reduce temperature gradients, which in turn helps prevent the evaporation of the propellant. In addition, the introduction of the cooled liquid also reduces the temperature of the bulk fluid. If the jet has a moderate amount of momentum, it can cause the formation of a geyser at the liquid-vapor interface. The increased area of the free surface due to the formation of a geyser would help promote condensation, thus reducing the pressure even further. At higher levels of jet momentum, the geyser will strike the opposite end of the tank and either form a separate pool or "roll" down the tank walls re-mixing with the bulk fluid. As the fluid comes around the tank walls there will be a cooling effect on the wall. However, it must be remembered that the addition of excessive kinetic energy to the bulk fluid will eventually result in undesirable heat generation through viscous dissipation. Therefore, it is necessary to classify the flow and predict its behavior to optimize the system.

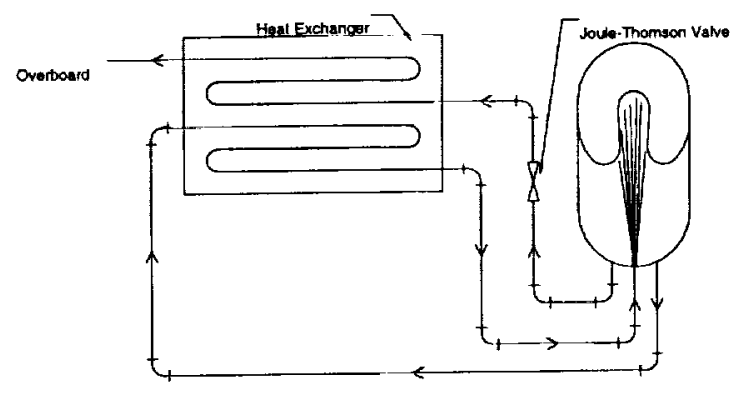

Fig. 1. Simple schematic of a TVS.

\section{Flow Patterns}

A morphology has been defined based on four distinct flow patterns that occur when an axial jet is injected into the bulk liquid region of a propellant tank in a microgravity environment ${ }^{1}$. These flow patterns are defined as follows and illustrated in Figure 2 with velocity fields from CFD simulations. These figures represent only half of the tank; the flow was modeled axisymmetrically.

I. Dissipation of the jet in the bulk liquid region.

II. Geyser Formation.

III. Collection of jet liquid in the aft end (opposite to the jet inlet) of the tank.

IV. Liquid circulation over the aft end of the tank and down the tank walls re-mixing with the bulk liquid. 


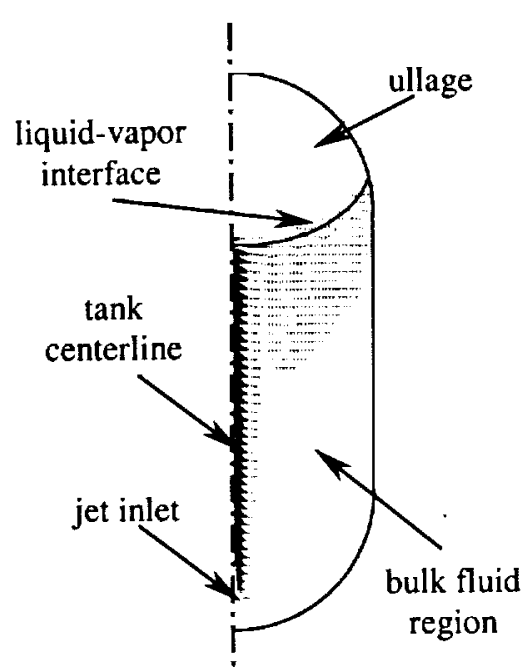

I

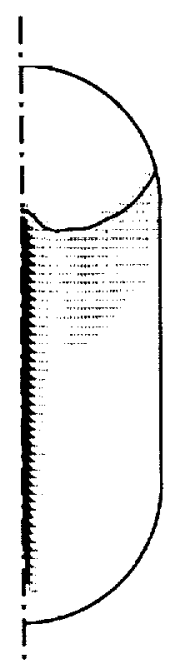

II

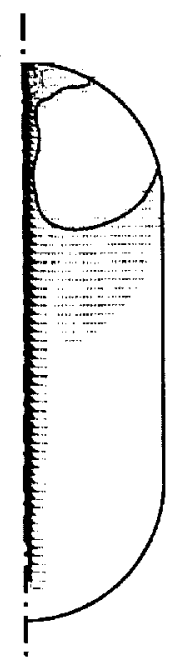

III

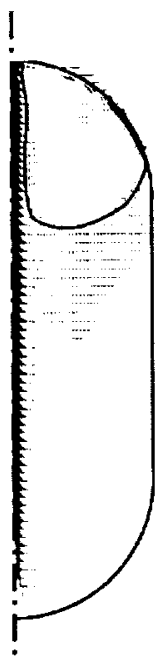

IV

Fig. 2. Jet flow patterns.

The ability to predict the flow pattern for any given combination of tank geometry, tank fill level, gravitational acceleration, and jet momentum is crucial for optimization and implementation of a TVS. A study of geysers and the development of an accurate tool for predicting their behavior are therefore necessary in the process of developing the TVS concept.

\section{COMPUTATIONAL MODEL}

The complications and expense of microgravity experimentation severely limits the amount of data that can be collected for microgravity geyser formation. Therefore, an attractive alternative is the use of computational fluid dynamics, or CFD, to model the geyser flows. The use of a CFD code can significantly reduce the cost of investigating the microgravity geyser phenomena. It also allows for easy manipulation of parameters such as tank size, fluid properties, jet momentum levels, and gravitational acceleration levels.

The ECLIPSE code was chosen for its previously demonstrated ability to model the microgravity geyser flows of interest here ${ }^{4,5}$. ECLIPSE is a modified version of the RIPPLE ${ }^{6}$ code developed at Los Alamos National Laboratory. RIPPLE was written to solve the general incompressible, two-dimensional Navier-Stokes equations. It is a descendent of the NASA VOF2D code, and was developed to model "transient, twodimensional, laminar, incompressible fluid flows with free surfaces of general topology." The flow field is discretized into finite volumes to form a non-uniform mesh. This non-uniform mesh allows the use of finer mesh in areas that may require more accuracy and coarser mesh in less critical areas. The code models free surfaces with volume of fluid (VOF) data on the mesh, and a continuum surface force (CSF) model is used to model surface tension. Staggered grid differential equation approximations result in a system of algebraic equations that are solved by a two step projection method employing an incomplete Cholesky conjugate gradient (ICCG) solution technique for the pressure Poisson equation (PPE) ${ }^{6}$. ECLIPSE has been enhanced with the addition of the two equation JonesLaunder $k-\varepsilon$ turbulence model with a Pope round jet correction term. A slight modification to the $k-\varepsilon$ model constant $C_{\varepsilon l}$ (changed from 1.4 to 1.6 ) was employed per Pope ${ }^{7}$. This was done to better account for the dissipation of turbulence kinetic energy in an axisymetric jet. A detailed account of the modeling methodology can be found in reference (8).

\section{DIMENSIONAL ANALYSIS}

The objective of the dimensional analysis is to establish a correlation for predicting geyser formation and geyser height without the expense of CFD. The correlation developed should be general enough to allow its implementation to any combination of tank size, fill level, working fluid, or acceleration level. The development of such a correlation has already been attempted, but like most models and correlations there is room for improvement.

\section{Established Correlations}

Through drop tower testing completed in the NASA LeRC 5 second zero gravity facility, Aydelott ${ }^{2}$ determined that inertia, acceleration, and surface 
tension forces are the primary factors affecting geyser formation in a microgravity environment. It was further deduced that the main parameters in the dimensional analysis should be the jet velocity, liquid density and surface tension, acceleration environment, geyser height, and selected characteristic lengths. Using the Buckingham Theorem, Aydelott established three dimensionless groups pertinent to predicting geysers and geyser height. These dimensionless groups are the jet-Bond number $B o$, the jet-Weber number $W e$, and a non-dimensional geyser height $G$.

The Bond number is the ratio of acceleration to surface tension forces, and the Weber number is the ratio of inertia to surface tension forces. Aydelott formed these dimensionless groups as follows:

$B o=\frac{a \rho R_{i}^{2}}{\sigma}=\frac{\text { acceleration force }}{\text { surface tension force }}$

$W e=\frac{\rho V_{0}^{2} R_{o}^{2}}{\sigma D,}=\frac{\text { inertia force }}{\text { surface tension force }}$

$G=\frac{h_{x}}{R_{r}}=\frac{\text { geyser height }}{\text { tank radius }}$

where $o$ indicates the jet at the pipe outlet, $j$ indicates the jet at the liquid-vapor interface, and $t$ indicates the tank. Also, note that $h_{g}$ is measured from the initial height of the free surface above the jet outlet. The jet radius at the liquid-vapor interface is calculated with an empirical correlation for completely turbulent jets dependent upon the liquid height above the jet outlet $h_{b}$.

$R_{j}=R_{\mathrm{o}}+0.12 h_{\mathrm{b}} \rightarrow h_{\mathrm{b}} \leq 12.4 R_{\circ}$

$R_{j}=0.11 R_{\mathrm{o}}+0.19 h_{\mathrm{b}} \rightarrow h_{ }>12.4 R_{\mathrm{o}}$

Aydelott concluded from his data that a linear relation existed between the non-dimensional geyser height and the Weber number. He also assumed that the nondimensional geyser height should be inversely proportional to the Bond number. That is, the less acceleration to "hold down" the fluid the larger the geyser. This led to a correlation of the following form:

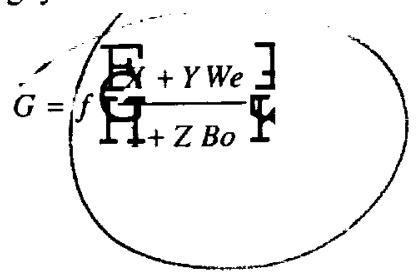

where $X, Y$, and $Z$ are experimentally determined constants. Through a least-squares curve fitting of experimental data, Aydelott formed the following correlation for completely turbulent jets:

$F=\frac{-0.5+1.6 W e}{1+0.6 B o}$

where $F$ is the flow characterization parameter and is expected to equal the dimensionless geyser height $G$. This correlation results in a root mean square (RMS) error of 0.25 between the experimental $G$ and $F$ data provided from the drop tower testing in reference (1).

The authors previously formulated a correlation using a combination jet/tank-Bond number ${ }^{9}$. Here the argument was that depending on the tank to jet diameter ratio, surface tension forces could play varying local and global effects. That is, before the onset of the jet, global effects are dominant as is visible by the large meniscus in the tank. However, once the jet impinges on the free surface, local effects near the geyser itself become quite important. The resulting Bond number and experimental data correlation using a least squares fit to Aydelott's data are as follows.

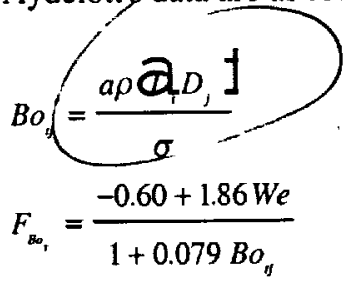

The result was a reduction of RMS error in geyser height prediction from 0.25 to 0.16 , or nearly a $35 \%$ reduction. Similar results were produced for computational data for the test tank as well as an enlarged tank.

\section{Improved Parameter Formulation}

Surface tension effects of both local and global scale are likely important in the formation of geysers.

Therefore, the mathematical model used to describe these local and global surface tension forces should include tank and jet dimensions. The previous use of a jet/tank bond number gives equal weight to both local and global effects. However, this may not be the case in the actual physics of the problem

In an effort to further the accuracy of the geyser correlation, the use of both a jet-Bond number and a tank-Bond number with separate weighting coefficients has been made. Also, the characteristic lengths have been taken to be the jet and tank diameters for consistency rather than a combination of diameters and 
radii. The following are the forms of the dimensionless parameters used in this study.

$$
\begin{aligned}
& \text { Non-Dimensional Geyser Height } \\
& G=\frac{h_{1}}{R_{r}}
\end{aligned}
$$

Jet-Bond Number

$$
B o_{,}=\frac{a \rho D_{i}^{2}}{\sigma}
$$

Tank-Bond Number

$$
B o_{r}=\frac{a \rho D_{1}^{2}}{\sigma}
$$

Jet-Weber Number

$$
W e=\frac{\rho V_{0}^{2} D_{0}^{2}}{\sigma D_{1}}
$$

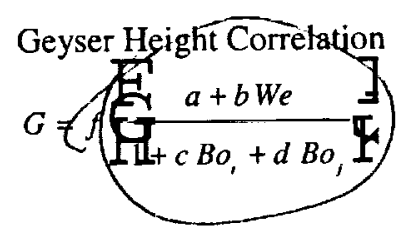

Several combinations of the two Bond numbers were investigated. These included both $2^{\text {nd }}$ and $3^{\text {rd }}$ order Bond number terms. Although the higher order forms improved the prediction accuracy of the correlation, they were dismissed. The small number of data points used to form theses correlation raised doubt if the higher order versions were actual improvements or were custom tailoring the correlation in inappropriate ways to fit the limited set of data points. Thus, the version shown in equation (14) is used exclusively in this study.

\section{BASELINE CORRELATION}

A subset of the drop tower test data ${ }^{2}$ (completely turbulent jets in the cylindrical tank with hemispherical heads) was used to establish the coefficients in the new correlation, equation (14). The exact parameters of these cases can be found in the summary table at the end of this paper. The coefficients of the new correlation were determined using an optimization routine that minimized the sum of the squared errors between the measured geyser height and the correlation predicted geyser height. The new correlation results in the following form after the optimization fitting of these coefficients.

$$
F_{r t}=\frac{-0.9+0.65 W e}{1+0.022 B o_{1}+0.053 B o_{1}}
$$

With these 14 experimental data points from the drop tower testing, equation (15) results in a RMS error in geyser height prediction of 0.15 compared to 0.25 for Aydelott's original correlation. That is a $40 \%$ reduction of error.

Figure 3 presents the predicted geyser heights and gives a graphical representation of the new correlations improvement. Note that in this figure data lying on a line of slope equal to 1 would indicate an ideal correlation, that is $F$ precisely predicts $G$.

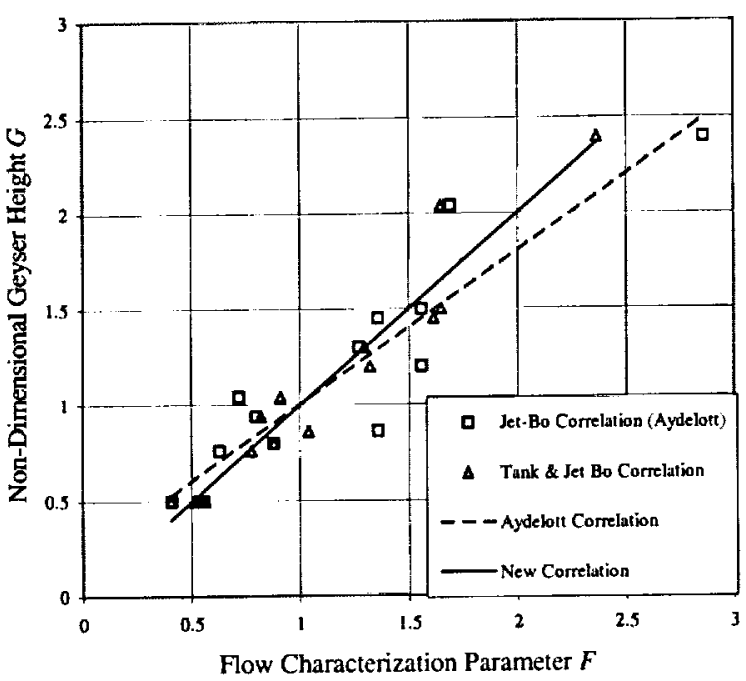

Fig. 3. Graphical comparison of the new geyser correlation, equation (15), to Aydelott's correlation, equation (7). All experimental data.

\section{Expanded Geyser Data Set}

Due to the limited amount of experiment data, 12 additional computational cases were added to the original drop tower test data. The cases were chosen to more evenly fill in the jet-Weber and jet-Bond number parameter space (Figure 4), and their exact parameters can be found in the summary table at the end of this paper. These cases were run using ECLIPSE and were combined with computational results of the original drop tower cases. The accuracy of both Aydelott's correlation and the new correlation were then compared using the RMS error method on the expanded data set. 


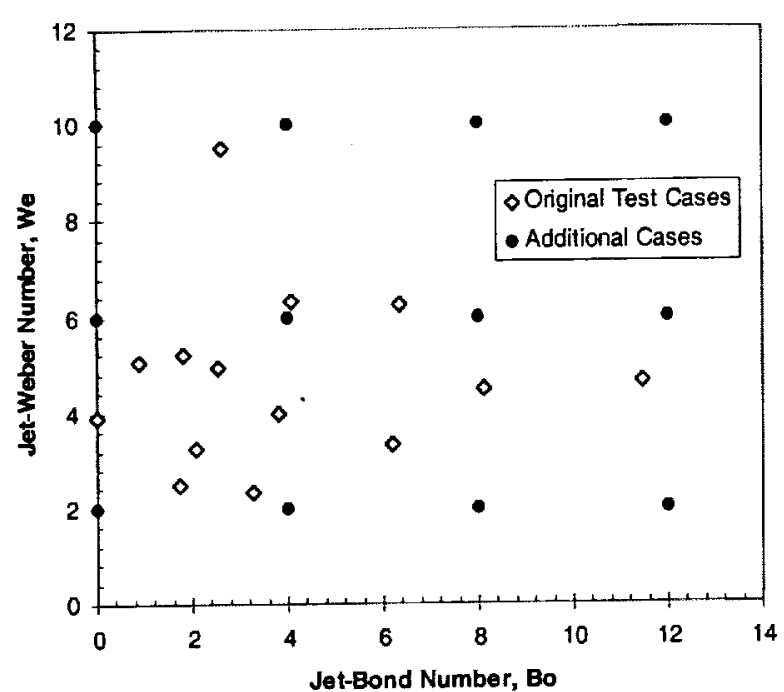

Fig. 4. Jet-Weber number and jet-Bond number parameter space.

The RMS errors for the new correlation and the Aydelott correlation are 0.26 and 0.34 respectively. That means that with the computational results, the new correlation yields nearly a $24 \%$ reduction in error over the original correlation. In addition, the new correlation predicted the two simulated flow pattern III or IV cases while the Aydelott correlation predicted only one. It is also noted that both correlations had one false flow pattern III or IV prediction. Figure 5 is a plot of the computed geyser heights $G$ verses the correlation predictions $F$ and shows that the new correlation gives less data spread from the linear trend than the Aydelott correlation. Again, note that a line of slope equal to one in this plot would represent an ideal correlation.

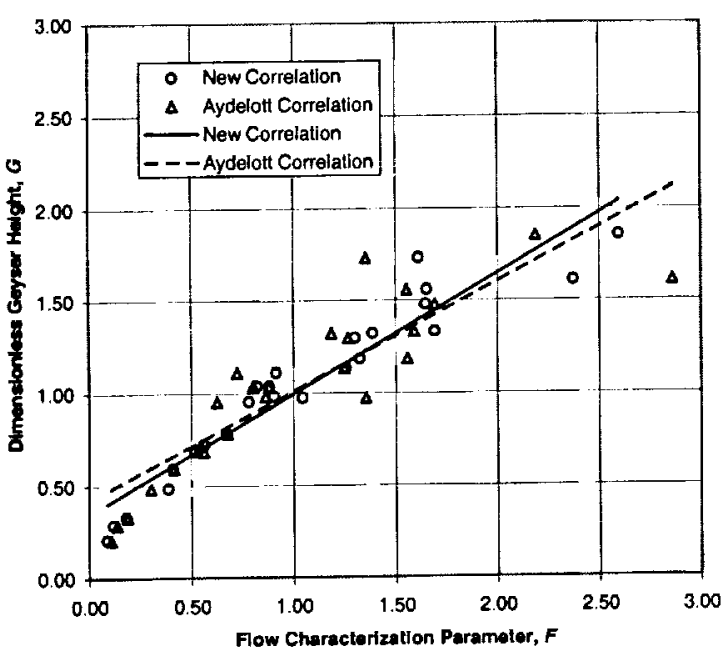

Fig. 5. Graphical comparison of the new geyser correlation to Aydelott's correlation. All computational data.
It must be noted that both the correlation developed here and Aydelott's correlation were developed using one small-scale tank using the same tank diameter to jet diameter ratio and test fluid. The applicability of the given correlations to large-scale tanks, different tank to jet diameter ratios, and other fluids is unknown. Thus, a study of these effects is in order. The ease of parameter manipulation and the cost effectiveness of CFD has been exploited here to further study these effects.

\section{TANK CONFIGURATIONS}

To determine the general applicability of the developed correlations to tanks of different geometries and scales, two additional tanks have been modeled. The original test tank used by Aydelott in the drop tower testing had a tank radius of $5 \mathrm{~cm}$ and an overall height of $20 \mathrm{~cm}$. The jet pipe was $0.2 \mathrm{~cm}$ in radius and protruded $I \mathrm{~cm}$ above the bottom of the tank. An augmented smallscale tank has been modeled and is a $50 \%$ wider version of Aydelott's tank with the same jet pipe. This makes a tank of $7.5 \mathrm{~cm}$ radius and an overall tank height of 25 $\mathrm{cm}$ resulting in an increase of tank to jet diameter ratio from $25: 1$ to $37.5: 1$. The full-scale tank is based on an orbit transfer vehicle (OTV) tank ${ }^{10}$. The OTV tank is 2 $\mathrm{m}$ in radius with an overall height of $10 \mathrm{~m}$. The jet pipe is $0.2 \mathrm{~m}$ long and $0.09 \mathrm{~m}$ in diameter. This gives a tank to jet diameter ratio of approximately 22:1. Figure 6 shows the dimensions and relative proportions of these tanks.
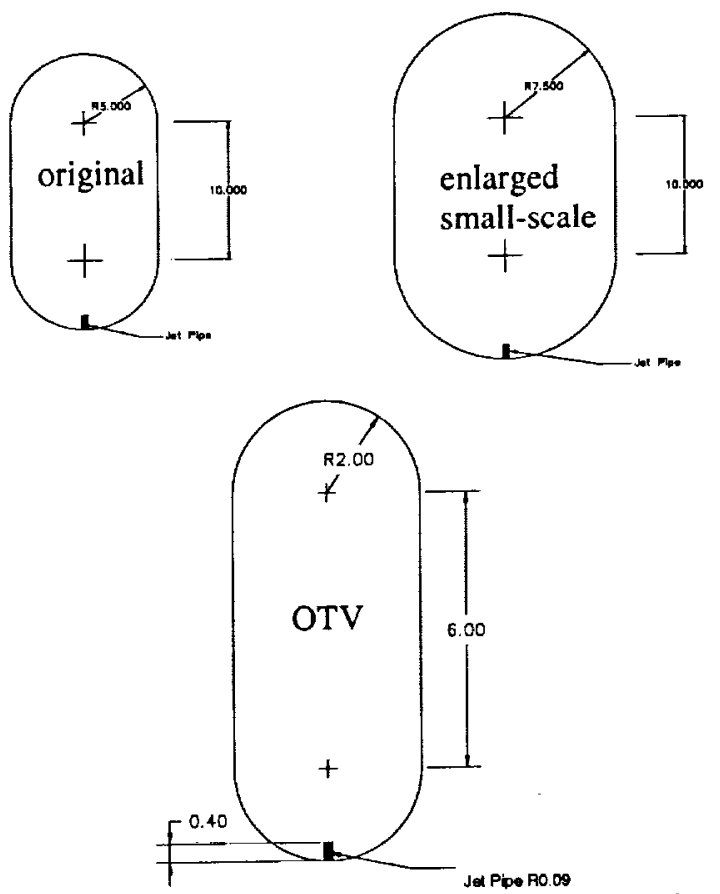

Fig. 6. Tank dimensions. Small-scale tank dimensions in centimeters and full-scale tank dimensions in meters. 
The test matrices chosen for the two tanks were formed to provide a regular distribution across the jet-Weber and jet-Bond number parameter space. The cases selected for the enlarged small-scale tank had the same jet-Bond and jet-Weber numbers of the original drop tower tests and the 12 addition cases discussed in the previous section. It should be noted that these parameters combined with the large diameter jet pipe of the OTV tank resulted in very low jet flow rates. Thus, the time required for the flows to reach a steady state condition were dramatically increased, but the required computational time step increased accordingly resulting in similar compute times. The OTV tank cases were performed with liquid hydrogen properties instead ethanol as was used in the drop tower and all previous simulations thus far.

\section{Small-Scale Tank}

The resulting RMS error from the 28 simulations of the enlarged small-scale tank indicates the new correlation, equation (15), provides a more accurate prediction of geyser height than the Aydelott correlation. The RMS errors for the new correlation and the Aydelott correlation are 0.36 and 0.64 respectively. It is also noted that both correlations predicted the one case that resulted in a flow pattern III or IV, as well as one false pattern III or IV prediction each. Figure 7 is a plot of the simulated geyser heights against the correlation predictions. Note that the plot reveals less data spread for the new correlation, thus the smaller RMS error value.

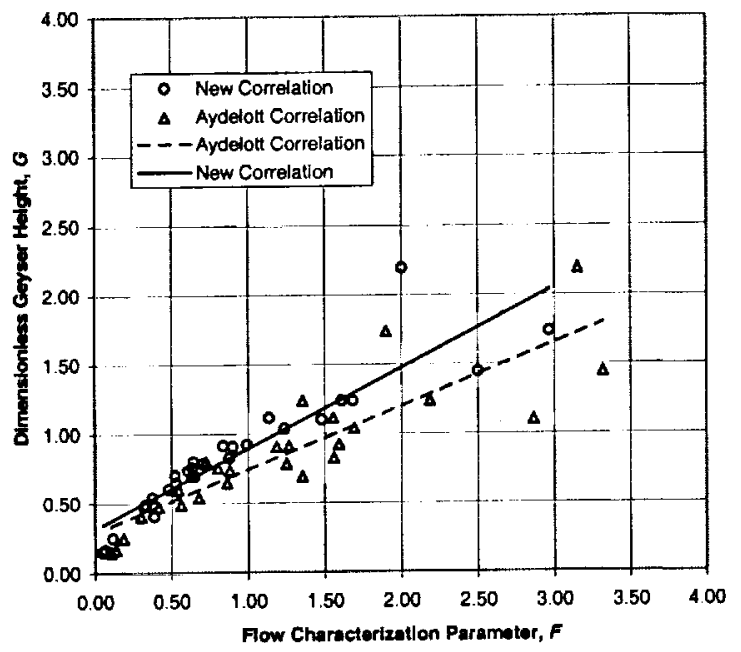

Fig. 7. Graphical comparison of the new geyser correlation, equation (15), to Aydelott's correlation, equation (7). All computational data for the enlarged small-scale tank.

\section{Full-Scale Tank}

The results from the 12 OTV tank simulations were much less favorable for the new correlation. The Aydelott correlation out predicted the new correlation with a RMS error of 0.16 compared to 0.34 . Although there is a relatively large difference, both errors are relatively small. However, there is no denying the new correlation's error is double that of Aydelott's correlation for the full-scale simulations. Both correlations predicted one of the two flow pattern III or IV cases with no false predictions. Figure 8 presents the results from these simulations compared with the correlation predictions.

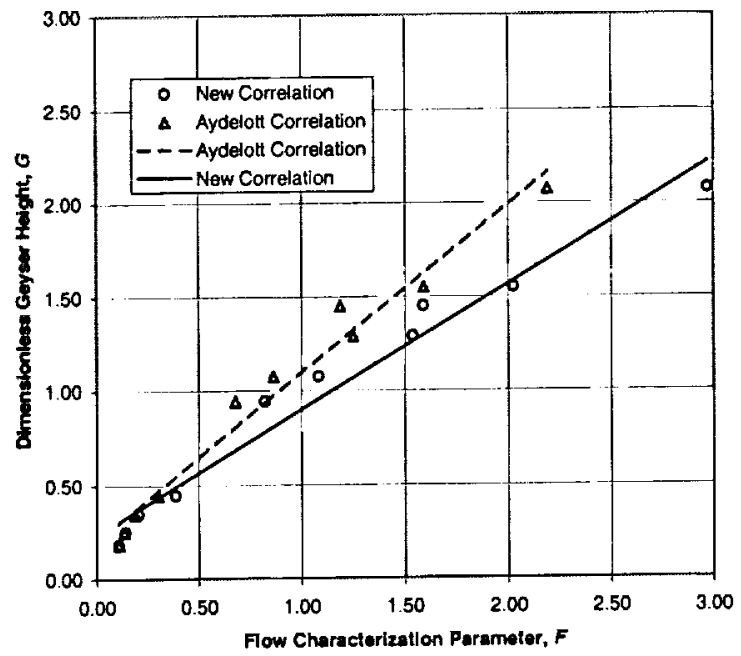

Fig. 8. Graphical comparison of the new geyser correlation, equation (15), to Aydelott's correlation, equation (7). All computational data for the OTV fullscale tank.

The objective of the work in this section was to determine the applicability of the developed correlations to tanks with different tank to jet diameter ratios, and to tanks of a much larger scale than the one used in developing the correlations. The results are somewhat mixed. The enlarged small-scale tank results showed that the new correlation, which includes tank scale Bond number effects, is a more accurate. Conversely, the full-scale OTV tank simulations produced RMS errors that indicate the new correlation was not as accurate as the Aydelott correlation.

It should be noted that the small-scale drop tower testing was limited to 5 seconds of reduced gravity. Part of this time was spent on initialization of the fluid free surface allowing it to come to its minimum energy meniscus configuration. This left the researcher with only 3.1 seconds of jet flow time. It is unclear from the literature whether the geysers in the drop tower tests 
were fully formed in the short 3.1 second run time. Some of the simulations performed here indicated that not all cases are fully formed. However, for consistency with the experiment, all data for the computational small-scale simulations was taken at 3.1 seconds. In addition, note that the OTV tank simulation data was taken at 1000 seconds, after the geyser was observed to be fully formed (this was necessary to allow the slow jet velocities enough time to form the geysers). Since the time scales of the simulations are quite different, the times the data were taken for the different scale tanks may not correspond to each other. Also, it would obviously be desirable to take data only once it is sure that the geyser is fully formed and not in a transient state.

One other possible reason for the mixed results in the tank configuration study is the inclusion of the tankBond number in the new correlation. The new correlation, equation (15), gives a weighting coefficient to each Bond number. These coefficients indicate the importance of local and global surface tension effects. Now recall that the OTV tank diameter is 40 times greater than that of the small-scale tank used to develop the correlation. It is possible that as the tank scale increases by such a magnitude the weighting factors for the two Bond numbers in equation (15) will change. If this is indeed the case, then the development of a correlation should at least include data from full-scale tanks of roughly the same scale as those to which the correlations will eventually be applied.

\section{LIOUID OXYGEN AND LIOUID HYDROGEN}

The geyser formation phenomena has been studied by various investigators since the 1970's. However, most of this investigation, experimental and computational, has been conducted using test fluids such as ethanol or freon instead of the actual cryogenic propellants used in propulsion systems. The reason for avoiding the cryogenic fluids stems from cost, handling, and safety issues. The use of CFD, however, allows us to safely and inexpensively investigate the geyser behavior in a computational environment with any fluid.

The most commonly used cryogenic propellant-oxidizer combination found in liquid propulsion systems is liquid hydrogen (LH2) and liquid oxygen (LOX). ECLIPSE has been used to simulate geyser formation with both of these fluids, and assess the accuracy of the two correlations discussed previously. Also, note that the full-scale simulations of the previous section were made with $\mathrm{LH} 2$ properties.
The CFD simulations with the cryogenic fluids have been conducted with the original drop tower test tank geometry. The test matrix consisted of the same 12 cases used to fill in the Weber-Bond number parameter space substituted with LOX and LH2 fluid properties.

\section{LOX Results}

The simulation results and correlation predictions of the LOX small-scale tank cases are presented in Figure 9.

The RMS errors for the new correlation and the Aydelott correlation equation are 0.24 and 0.19 respectively. Here the new correlation did not predict the geyser height as accurately as did the Aydelott correlation. However, the new correlation did predict all three flow pattern III or IV cases where the Aydelott correlation predicted only one. Neither correlation made any false pattern III or IV predictions.

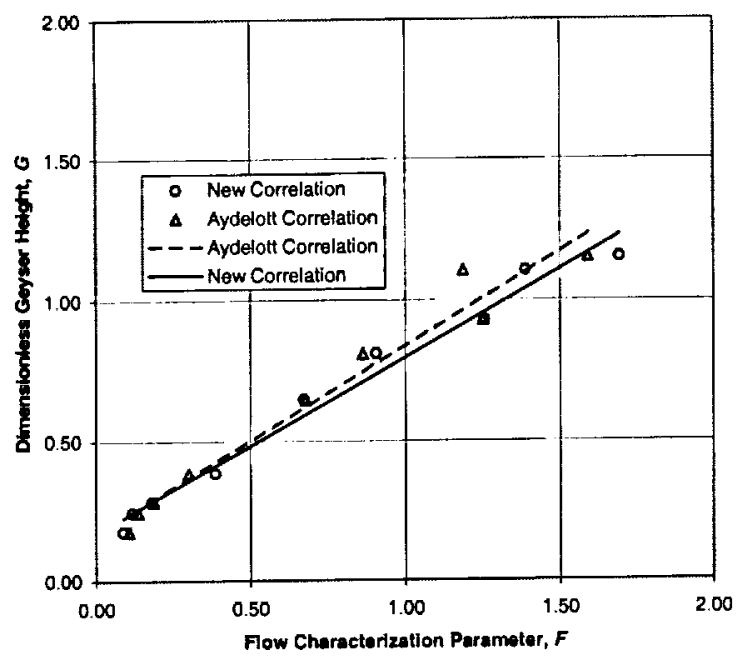

Fig. 9. Graphical comparison of the new geyser correlation, equation (15), to Aydelott's correlation, equation (7). All computational data with LOX.

\section{LH2 Results}

The new correlation faired somewhat better with the LH2 simulations, but was still slightly less accurate than the Aydelott correlation. Figure 10 present the results of these simulations. The resulting RMS errors for the new correlation and the Aydelott correlation are 0.18 and 0.14 respectively. Thus, Aydelott's correlation is more accurate, but not by a real significant amount. This is seen in the similarity of the data spread in Figure 10. Three simulations produced a flow pattern III or IV with LH2. Out of these three cases, the new correlation predicted all three while the Aydelott correlation predicted only two. Neither correlation made any false flow pattern III or IV predictions for the LH2 cases. It should also be noted that the calculation 
for case "w2p5b2" did not converge to a solution leaving a hole in the test matrix at this point. The reason for this miscalculation was not determined.

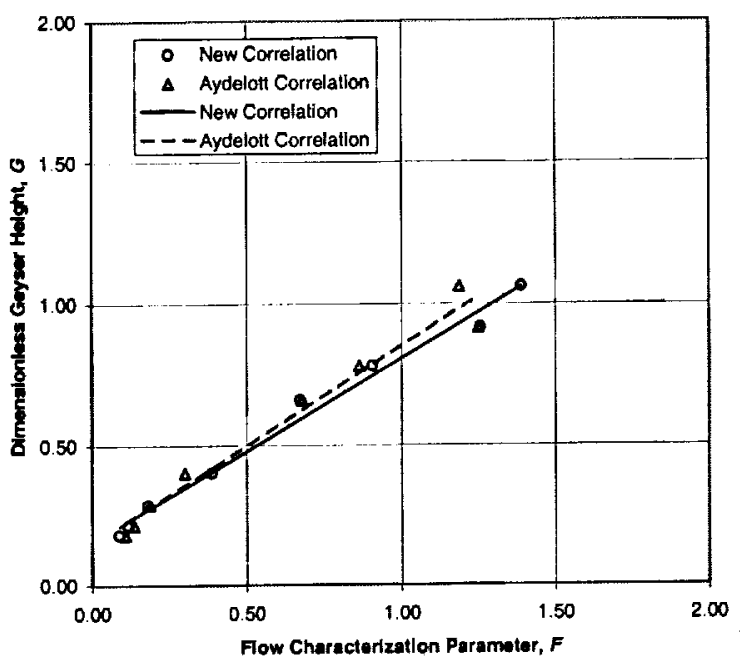

Fig. 10. Graphical comparison of the new geyser correlation, equation (15), to Aydelott's correlation, equation (7). All computational data with LH2.

In the cases using LOX and LH2 as the working fluids the new correlation did not produce RMS errors that were improvements over the Aydelott correlation. However, the new correlation did show a superior ability to predict the occurrence of flow pattern III/TV. While absolute geyser height accuracy would be preferable, the implementation of a TVS may be more concerned with flow pattern prediction than precise geyser height. Thus, these results still show merit for the new correlation.

It should also be remembered that the correlations that have been studied were developed from a rather small set of experimental data. To improve the confidence in either correlation, more data should be gathered and used in their formulation.

\section{REFINED CORRELATIONS}

The correlations that have been applied and investigated so far were developed using the limited experimental test data from a drop tower. In the present study, a substantial amount of computational data has been collected through simulations with ECLIPSE. This data has been used to refine the coefficients in each correlation, equations (7) and (15). The RMS errors from these refined correlations should give a measure for the overall geyser height predictive accuracy of each correlation method.
Results

Equations (16) and (17) are the results of the optimization of the correlation coefficients for the new correlation and the Aydelott correlation respectively. The RMS errors for the new and Aydelott correlations were 0.157 and 0.242 respectively. Note that the Aydelott correlation was formed using his definitions of Bond and Weber numbers. Figure 11 is a plot of all geyser data, experimental and computational, versus the refined correlation predictions. These refined correlations indicate that the new method using both tank and jet-Bond numbers is superior to Aydelott's correlation that uses only the jet-Bond number.

$$
\begin{aligned}
& F_{T H}=\frac{-0.012+0.316 \mathrm{We}}{1+0.013 B o_{1}+0.0134 B o_{1}} \\
& F_{A V D}=\frac{0.19+0.72 W e}{1+0.29 B o}=
\end{aligned}
$$

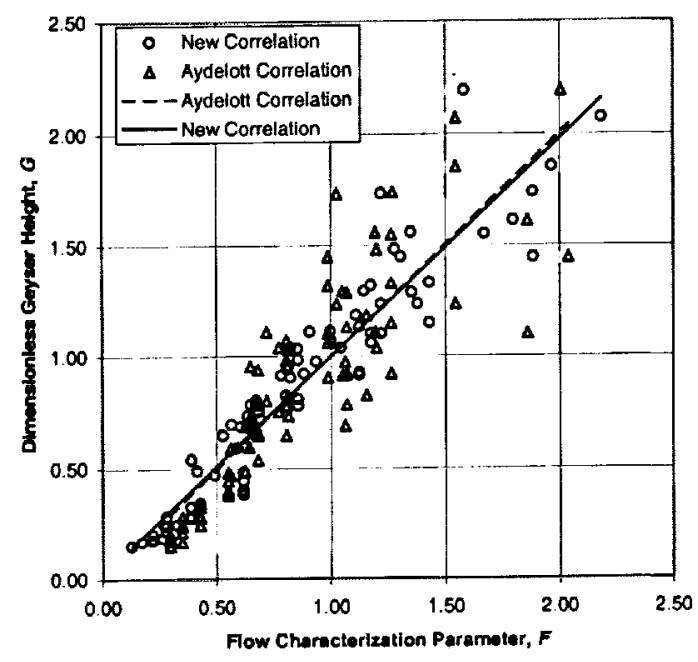

Fig. 11. Graphical comparison of the refined correlations, equations (16) and (17). All data computational and experimental.

\section{SUMMARY AND CONCLUSIONS}

Although not seen in every subset of data analyzed, it appears to be the overall conclusion that the new correlation developed in this study using both tank and jet-Bond numbers is the superior correlation. Thus, the global surface tension effects discussed previously do appear to be pertinent in describing microgravity geyser behavior. At this point the new corrleation refined with the full data set of this study, equation (16), is the most 
accurate known method available to predict microgravity propellant tank geysers short of a full CFD analysis. It is recommended that this correlation be used to design further geyser experiments as well as in preliminary design studies of a Thermodynamic Vent System.

It also appears that the application of any correlation to predict geysers in full-scale tanks will need to be formed with accurate data obtained from tanks of a similar scale. Thus, the continued use and advancement of CFD for geyser flows will be necessary due to the size and cost of large-scale hardware testing. It is recommended that before a correlation is applied to the final design of a TVS, more data be collected for various full-scale configurations and the above correlations compared and refined further.

Table 1. Case parameter summary table.

\begin{tabular}{|c|c|c|c|c|c|c|}
\hline & Case & $\begin{array}{l}\text { Liquid Height } \\
\text { Above Jet Outlet, } \\
h_{b}(\mathrm{~cm})\end{array}$ & $\begin{array}{l}\text { Jet Reynolds } \\
\text { Number, Re }\end{array}$ & $\begin{array}{l}\text { Jet Weber } \\
\text { Number, We }\end{array}$ & $\begin{array}{l}\text { Jet Bond } \\
\text { Number, Bo }\end{array}$ & $\begin{array}{l}\text { Non-Dimensional } \\
\text { Geyser Height, G }\end{array}$ \\
\hline \multirow{14}{*}{ 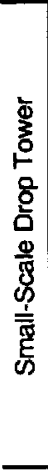 } & 6 & 5.8 & 1290 & 5.05 & 0.87 & 2.04 \\
\hline & 7 & 5.9 & 1320 & 5.20 & 1.79 & 1.20 \\
\hline & 8 & 5.9 & 1290 & 4.96 & 2.53 & 0.86 \\
\hline & 11 & 6.0 & 1800 & 9.51 & 2.61 & 2.40 \\
\hline & 24 & 7.9 & 1320 & 3.90 & 0 & 1.45 \\
\hline & 25 & 9.5 & 1320 & 3.25 & 2.08 & 0.94 \\
\hline & 26 & 9.1 & 1430 & 3.98 & 3.82 & 0.80 \\
\hline & 27 & 9.3 & 1320 & 3.32 & 6.20 & 0.50 \\
\hline & 31 & 9.3 & 1820 & 6.31 & 4.07 & 1.50 \\
\hline & 32 & 9.4 & 1820 & 6.25 & 6.33 & 1.30 \\
\hline & 58 & 12.4 & 1320 & 2.50 & 1.72 & 0.50 \\
\hline & 59 & 12.2 & 1270 & 2.35 & 3.26 & 0.50 \\
\hline & 61 & 12.9 & 1800 & 4.47 & 8.12 & 1.04 \\
\hline & 62 & 12.7 & 1820 & 4.64 & 11.48 & 0.76 \\
\hline \multirow{26}{*}{ 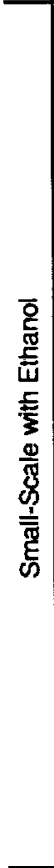 } & 6 & 5.8 & 1290 & 5.05 & 0.87 & 1.48 \\
\hline & 7 & 5.9 & 1320 & 5.20 & 1.79 & 1.19 \\
\hline & 8 & 5.9 & 1290 & 4.96 & 2.53 & 0.98 \\
\hline & 11 & 6.0 & 1800 & 9.51 & 2.61 & 1.61 \\
\hline & 24 & 7.9 & 1320 & 3.90 & 0 & 1.73 \\
\hline & 25 & 9.5 & 1320 & 3.25 & 2.08 & 1.04 \\
\hline & 26 & 9.1 & 1430 & 3.98 & 3.82 & 1.04 \\
\hline & 27 & 9.3 & 1320 & 3.32 & 6.20 & 0.69 \\
\hline & 31 & 9.3 & 1820 & 6.31 & 4.07 & 1.56 \\
\hline & 32 & 9.4 & 1820 & 6.25 & 6.33 & 1.30 \\
\hline & 58 & 12.4 & 1320 & 2.50 & 1.72 & 0.72 \\
\hline & 59 & 12.2 & 1270 & 2.35 & 3.26 & 0.59 \\
\hline & 61 & 12.9 & 1800 & 4.47 & 8.12 & 1.11 \\
\hline & 62 & 12.7 & 1820 & 4.64 & 11.48 & 0.96 \\
\hline & wp5bo & 9 & 951 & 2 & 0 & 0.48 \\
\hline & wp5b1 & 9 & 951 & 2 & 4 & 0.33 \\
\hline & wp5b2 & 9 & 951 & 2 & 8 & 0.28 \\
\hline & wp5b3 & 9 & 951 & 2 & 12 & 0.21 \\
\hline & w1p5bo & 9 & 1647 & 6 & 0 & * \\
\hline & w1p5b1 & 9 & 1647 & 6 & 4 & 1.32 \\
\hline & w1p5b2 & 9 & 1647 & 6 & 8 & 0.98 \\
\hline & w1p5b3 & 9 & 1647 & 6 & 12 & 0.78 \\
\hline & w2p5b0 & 9 & 2127 & 10 & 0 & * \\
\hline & w2p5b1 & 9 & 2127 & 10 & 4 & 1.85 \\
\hline & w2p5b2 & 9 & 2127 & 10 & 8 & 1.33 \\
\hline & w2p5b3 & 9 & 2127 & 10 & 12 & 1.13 \\
\hline
\end{tabular}




\begin{tabular}{|c|c|c|c|c|c|c|}
\hline & Case & $\begin{array}{l}\text { Liquid Height } \\
\text { Above Jet Outlet, } \\
h_{b}(\mathrm{~cm})\end{array}$ & $\begin{array}{c}\text { Jet Reynolds } \\
\text { Number, Re }\end{array}$ & $\begin{array}{l}\text { Jet Weber } \\
\text { Number, We }\end{array}$ & $\begin{array}{l}\text { Jet Bond } \\
\text { Number, Bo }\end{array}$ & $\begin{array}{l}\text { Non-Dimensional } \\
\text { Geyser Height, G }\end{array}$ \\
\hline \multirow{26}{*}{ 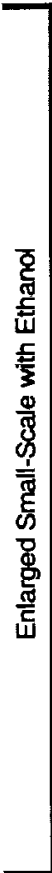 } & 6 & 5.8 & 1290 & 5.05 & 0.87 & 1.04 \\
\hline & 7 & 5.9 & 1320 & 5.20 & 1.79 & 0.82 \\
\hline & 8 & 5.9 & 1290 & 4.96 & 2.53 & 0.69 \\
\hline & 11 & 6.0 & 1800 & 9.51 & 2.61 & 1.45 \\
\hline & 24 & 7.9 & 1320 & 3.90 & 0 & 1.24 \\
\hline & 25 & 9.5 & 1320 & 3.25 & 2.08 & 0.75 \\
\hline & 26 & 9.1 & 1430 & 3.98 & 3.82 & 0.73 \\
\hline & 27 & 9.3 & 1320 & 3.32 & 6.20 & 0.49 \\
\hline & 31 & 9.3 & 1820 & 6.31 & 4.07 & 1.11 \\
\hline & 32 & 9.4 & 1820 & 6.25 & 6.33 & 0.91 \\
\hline & 58 & 12.4 & 1320 & 2.50 & 1.72 & 0.60 \\
\hline & 59 & 12.2 & 1270 & 2.35 & 3.26 & 0.47 \\
\hline & 61 & 12.9 & 1800 & 4.47 & 8.12 & 0.80 \\
\hline & 62 & 12.7 & 1820 & 4.64 & 11.48 & 0.70 \\
\hline & wp5b0 & 9 & 951 & 2 & 0 & 0.41 \\
\hline & wp5b1 & 9 & 951 & 2 & 4 & 0.25 \\
\hline & wp5b2 & 9 & 951 & 2 & 8 & 0.17 \\
\hline & wp5b3 & 9 & 951 & 2 & 12 & 0.15 \\
\hline & w1p5b0 & 9 & 1647 & 6 & 0 & 1.74 \\
\hline & w1p5b1 & 9 & 1647 & 6 & 4 & 0.91 \\
\hline & w1p5b2 & 9 & 1647 & 6 & 8 & 0.65 \\
\hline & w1p5b3 & 9 & 1647 & 6 & 12 & 0.54 \\
\hline & w2p5b0 & 9 & 2127 & 10 & 0 & * \\
\hline & w2p5b1 & 9 & 2127 & 10 & 4 & 1.24 \\
\hline & w2p5b2 & 9 & 2127 & 10 & 8 & 0.92 \\
\hline & w2p5b3 & 9 & 2127 & 10 & 12 & 0.78 \\
\hline \multirow{12}{*}{ 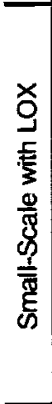 } & wp5b0 & 9 & 5539 & 2 & 0 & 0.38 \\
\hline & wp5b1 & 9 & 5539 & 2 & 4 & 0.28 \\
\hline & wp5b2 & 9 & 5539 & 2 & 8 & 0.24 \\
\hline & wp5b3 & 9 & 5539 & 2 & 12 & 0.17 \\
\hline & wtp5bo & 9 & 9594 & 6 & 0 & • \\
\hline & w1p5b1 & 9 & 9594 & 6 & 4 & 1.10 \\
\hline & w1p5b2 & 9 & 9594 & 6 & 8 & 0.81 \\
\hline & w1p5b3 & $\theta$ & 9594 & 6 & 12 & 0.65 \\
\hline & w2p5b0 & 9 & 12386 & 10 & 0 & " \\
\hline & w2p5b1 & 9 & 12386 & 10 & 4 & * \\
\hline & w2p5b2 & 9 & 12386 & 10 & 8 & 1.15 \\
\hline & w2p5b3 & 9 & 12386 & 10 & 12 & 0.93 \\
\hline \multirow{12}{*}{ 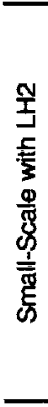 } & wp5bo & 9 & 8252 & 2 & 0 & 0.40 \\
\hline & wp5b1 & 9 & 8252 & 2 & 4 & 0.29 \\
\hline & wp5b2 & 9 & 8252 & 2 & $B$ & 0.21 \\
\hline & wp5b3 & 9 & 8252 & 2 & 12 & 0.18 \\
\hline & w1p5bo & 9 & 14293 & 6 & 0 & $\cdot$ \\
\hline & w1p5b1 & 9 & 14293 & 6 & 4 & 1.06 \\
\hline & w1p5b2 & 9 & 14293 & 6 & 8 & 0.78 \\
\hline & w1p5b3 & 9 & 14293 & 6 & 12 & 0.66 \\
\hline & w2p5bo & 9 & 18452 & 10 & 0 & * \\
\hline & w2p5b1 & 9 & 18452 & 10 & 4 & * \\
\hline & w2p5b2 & 9 & 18452 & 10 & 8 & did not converge \\
\hline & w2p5b3 & 9 & 18452 & 10 & 12 & 0.92 \\
\hline \multirow{12}{*}{ 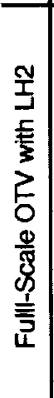 } & wp5bo & 400 & 53642 & 2 & 0 & 0.45 \\
\hline & wp5b1 & 400 & 53642 & 2 & 4 & 0.35 \\
\hline & wp5b2 & 400 & 53642 & 2 & 8 & 0.25 \\
\hline & wp5b3 & 400 & 53642 & 2 & 12 & 0.18 \\
\hline & w1p5bo & 400 & 92910 & 6 & 0 & $*$ \\
\hline & w1p5b1 & 400 & 92910 & 6 & 4 & 1.45 \\
\hline & w1p5b2 & 400 & 92910 & 6 & 8 & 1.07 \\
\hline & w1p5b3 & 400 & 92910 & 6 & 12 & 0.94 \\
\hline & w2p5bo & 400 & 119946 & 10 & 0 & • \\
\hline & w2p5b1 & 400 & 119946 & 10 & 4 & 2.07 \\
\hline & w2p5b2 & 400 & 119946 & 10 & 8 & 1.55 \\
\hline & w2p5b3 & 400 & 119946 & 10 & 12 & 1.29 \\
\hline
\end{tabular}

* Flow Pattern III/IV

11

American Institute of Aeronautics and Astronautics 


\section{REFERENCES}

1. Aydelott, J.C., "Axial Jet Mixing of Ethanol In Cylindrical Containers During Weightlessness," NASA TP-1487, 1979.

2. Aydelott, J.C., "Modeling of Space Vehicle Propellant Mixing,"NASA TP-2107, 1983.

3. Poth, L.J., and Van Hook, J.R., "Control of the Thermodynamic State of Space-Stored Cryogens by Jet Mixing," Journal of Spacecraft, Vol. 9, No. 5, May 1972, pp. 332-336.

4. Hochstein, J.I., Gerhart, P.M., and Aydelott, J.C., "Computational Modeling of Jet Induced Mixing of Cryogenic Propellants in Low-G," AIAA Paper 84-1344, 1984.

5. Wendl, J.C., Hochstein, J.I., and Sasmal, G.P., "Modeling of Jet Induced Geyser Formation in a Reduced Gravity Environment," AIAA Paper 91 0801, 1991.

6. Kothe, D.B., Mjolsness, R.C., and Torrey, M.D., "Ripple: A Computer Program for Incompressible Flow with Free Surfaces," Los Alamos National Laboratory, LA-12007-MS, 1991.

7. Pope S.B., "An Explanation of the Turbulent Round Jet/Plane Jet Anomaly," AIAA Journal, Vol. 16, No. 3, March 1978, pp. 279-281.

8. Thornton, R.J., "A Computational and Dimensional Analysis of Microgravity Propellant Tank Geyser Prediction," Magisterial Thesis, The University of Memphis, Memphis, Tennessee, 2000.

9. Thornton, R.J., and Hochstein, J.I., "Microgravity Geyser and Flow Field Prediction," AIAA Paper 00-0858, 2000.

10. Hochstein, J.I., Gerhart, P.M., and Aydelott, J.C., "Computational Modeling of Jet Induced Mixing of Cryogenic Propellants in Low-G," AIAA Paper 84-1344, 1984. 